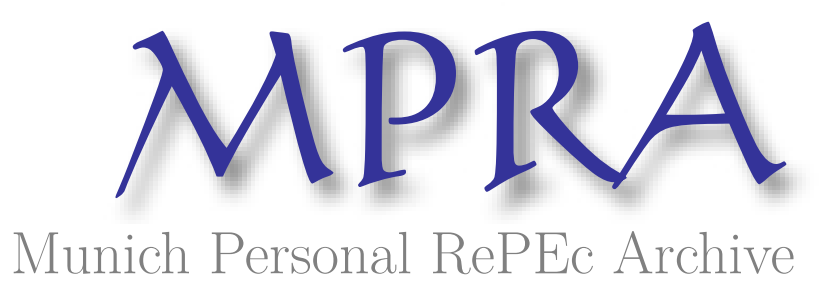

\title{
Forecasting The Pricing Kernel of IBNR Claims Development In Property-Casualty Insurance
}

Cadogan, Godfrey

DeLinc Research

10 June 2010

Online at https://mpra.ub.uni-muenchen.de/23235/

MPRA Paper No. 23235, posted 12 Jun 2010 11:33 UTC 


\title{
Forecasting The Pricing Kernel of IBNR Claims Development In Property-Casualty Insurance
}

\author{
Godfrey Cadogan * \\ Working Paper
}

June 11, 2010

${ }^{*}$ Corresponding address: 3401-B N.W. 72nd Ave, Ste \# T-419, Miami, FL 33122; e-mail: gocadog@gmail.com. I thank Mark Zanecki from ihaconsultants.com for introducing me to this problem. The usual disclaimer applies. 


\begin{abstract}
A new method of forecasting the pricing kernel, i.e., stochastic claim inflation or link ratio function, of incurred but not reported (IBNR) claims (in property-casualty insurance) from residuals in a dynamic claims forecast model is presented. We employ a pseudo Kalman filter approach by using claims risk exposure estimates to reconstruct innovations in stochastic claims development. Whereupon we find that the pricing kernel forecast is a product measure of the innovations. We show how these results impact performance measurement including but not limited to risk-adjusted return on capital by and through insurance accounting relationships for adjusted underwriting results; and loss ratio or pure premium calculations. Additionally, we show how, in the context of Wold decomposition, diagnostics from our model can be used to compute signal to noise ratio for, and cross check, unobservable pricing kernels used to forecast claims. Furthermore, we prove that a single risk exposure factor connects seemingly unrelated specifications for loss link ratio, and claims volatility.
\end{abstract}

Keywords: IBNR claims ladder; claims reserve forecast; stochastic claim inflation; claims risk exposure; link ratio function; property-casualty insurance; insurance accounting

JEL Classification Codes: C53, G22, G28, M49 


\section{Contents}

1 Introduction 2

2 IBNR Claim Ladder Model 3

3 Stochastic Claim Development 5

3.1 Detrended cumulative claims . . . . . . . . . . . . 5

3.2 Unobservable pricing kernel or link ratio function . . . . . . . . 6

4 Econometric specification of stochastic claims risk $\quad 7$

4.1 Pricing kernel estimator . . . . . . . . . . . . . . . . . . 12

4.1.1 Wold decomposition of pricing kernel . . . . . . . . 14

5 Insurance accounting and performance measurement 15

6 Conclusion $\quad 16$

$\begin{array}{lr}\text { References } & 18\end{array}$

\section{List of Figures}

1 IBNR Cumulative Claims Ladder . . . . . . . . . . . . . . . . 4 


\section{Introduction}

Claims reserve pricing is one of the most important elements of an insurance company's balance sheet. See (Küppelberg and Severin, 2001, pg. 1). In fact, according to (Ward and Lee, 2002, pg. 81) "Insurers bear a responsibility both to shareholders and policyholders to maintain solvency throughout a variety of potential adverse events." Consequently, risk management including but not limited to forecasts of claims development is part of that responsibility ${ }^{1}$. This paper's contribution to the literature on claims development and or claims reserve pricing theory stems from its provision of a MLE estimator for stochastic claims inflation with risk factors derived from claims risk exposure. Because stochastic claims inflation provides an unobservable nexus between incurred but not reported (IBNR) claims our result(s) should be of interest to practitioners as well ${ }^{2}$.

An important arsenal in risk management weaponry is stochastic claims volatility forecast, and of necessity, claims volatility modeling. For instance, Prof. Erhard Kremer presented a paper on stochastic claim inflation at the "1998 General Insurance Convention and ASTIN Colloquium" in Glasgow, Scotland during which he "... basically assumed that the discounted claims increase follows an autoregressive model of ARCH-type and that the stochastic yearly interest follows a classical autoregressive model," (emphasis added) (Kremer, 1999, pg. 602).

${ }^{1}$ Calandro and O`Brein (2004) described it thus:

Estimating the value of future claims is challenging for a number of reasons. First, future accident rates are unknowable, so attempts to predict them are inherently prone to error. Second, bodily injury and property damage claim values are extremely subjective, and many diverse factors ultimately determine claim values. Fault apportionment, the nature and extent of medical treatment and a persons responsiveness to that treatment, property repair or replacement considerations, attorney skill levels and skill sets, venue volatility, judicial objectivity, and quantification of an individuals pain and suffering, for example, all have a part to play in the claim valuation process.

$* * * * * * * * *$

Claim reserves represent a critical performance variable ... that must be implemented successfully for the intended strategy of the business to succeed. For example, under-reserving means equity is overstated, allowing an insurer to write more business than it can actually support. Worse, claim reserves are a large part of estimated historical losses, and are thus used in future rate making so under-reserving causes rate inadequacy, compounding the effect, and thus exacerbating the probability of insolvency. Over-reserving, on the other hand, may get an insurer a higher credit rating, but at the expense of offering high-priced insurance policies and at the risk of overpaying claims.

${ }^{2}$ For instance, some empirical research found that management discretion over claims loss accrual and reporting has been used to avoid reporting losses. Nelson (2000); Beaver et al. (2003). See (Shapland, 2007, pg. 120) for brief description of Statement of Statutory Accounting Practice (SSAP), and Generally Accepted Accounting Practice (GAAP) as it pertains to this issue. 
See also, (Wilkie, 1995, pg. 799). In fact, (Engle, 2001, pg. 158) plainly states that the goal of ARCH/GARCH models "... is to provide a volatility measurelike a standard deviation-that can be used in financial decisions concerning risk analysis, portfolio selection, and derivatives pricing" (emphasis added). En route to constructing our stochastic claims inflation estimator, we provide theoretical justification for Kremer and Wilkie's assumptions for stochastic claims growth, and ARCH-type modeling, by establishing a nexus between seemingly unrelated methodologies by Mack (1994) and De Jong (2006).

The rest of the paper proceeds as follows. In section 2 we provide a brief review of the canonical claim ladder or run off triangle model for IBNR claims reserving used to motivate results. In section 3 we introduce a stochastic claim development model based on detrended cumulative claims. Moreover, section 4 introduces an econometric specification for stochastic claims risk. The main result of the paper is reported in Theorem 4.6, and some heuristics on Wold decomposition of the pricing kernel is provided. In section 5 we provide a brief description of the impact of claims reserve pricing on insurance accounting and risk adjusted return on capital. In section 6 we conclude with perspectives.

\section{IBNR Claim Ladder Model}

In this section we briefly describe the salient charateristics of the so called Claim Ladder model for incurred but not reported claims. This section draws heavily on (Behncke, 2000, Ch. 11.4). See also, Taylor (1977).

Let $S_{i, j}$ be the incremental claim incurred in period $i$, developed in period $j-1^{3}$. Suppose that all developed claims are exhausted in period $n-1$. So that for each year $i$ the cumulative claim developed in period $j-1$ is

$$
C_{i, j}=S_{i, 1}+S_{1,2}+\ldots+S_{i, j}, \quad 1 \leq j \leq n
$$

The actuary's objective is to provide forecasts for $C_{i, j}$ in the face of claims trend. Thus, [s] he want to have a reserve $R_{i, j}$ on hand to satisfy claims as they develop in the future. Hence

$$
R_{i, j}=\hat{C}_{i, j}
$$

where $\hat{C}_{i, j}$ is claims forecast. Claims are developed according to the triangular

\footnotetext{
${ }^{3}$ The "time" $j$ occurs at the end of the period $j-1$.
} 


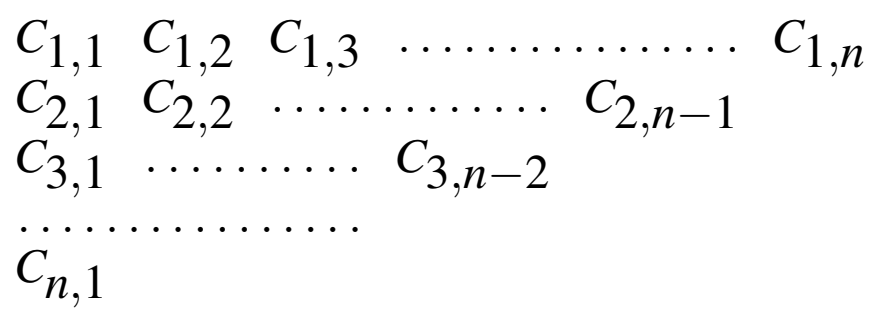

Figure 1: IBNR Cumulative Claims Ladder

pattern in Figure 1. Let $f_{j}$ be a stochastic inflation factor for claims developed in period $j-1$. In the asset pricing literature $f_{j}$ is defined as a pricing kernel or stochastic discount factor ${ }^{4}$. So that claims are linked as follows

$$
C_{i, j+1}=f_{j} C_{i, j}
$$

We make the following assumptions.

Assumption 2.1. There are no catastrophic claims in the model.

Assumption 2.2. Claims $C_{1, j}, C_{2, j}, \ldots, C_{n, j}$ are year wise independent.

Assumption 2.3. The pricing kernel $f_{j}$ is independent of the period $i$ when claims incurred.

Assumption 2.4. All claims are for the same line of business and are developed under the same regime.

Assumption 2.5. $\lim _{k \rightarrow \infty} f_{k}=1$

Let $\Omega$ be a sample corresponding to the laws of nature (according as they apply over the duration of claims development), $P$ be a probability measure on $\Omega$, and $\mathscr{F}$ be a $\sigma$-field of Borel subsets of $\Omega$. We designate the filtration of $\sigma$-fields $\mathscr{F}_{j} \subseteq$ $\mathscr{F}_{k}, \quad 0 \leq s \leq t$ by $\mathbb{F}$. The $\sigma$-field $\mathscr{F}_{0}$ contains the P-null sets of $\mathbb{F}$. Thus, our model is developed on the filtered probability space $(\Omega, \mathscr{F}, \mathbb{F}, P)$. In the interest of notational economy we suppress the " $\omega$ " notation unless otherwise indicated. Based on the foregoing assumptions, we rewrite Equation 2.3 as

$$
E\left[C_{i, j+1} \mid \mathscr{F}_{j}\right]=f_{j} C_{i, j}
$$

The recursive nature of Equation 2.3 and Equation 2.4 together with the claims

\footnotetext{
${ }^{4}$ See (Campbell et al., 1997, pp. 294-296) for definition and discussion of this concept.
} 
triangle shows that if all claims are developed by period $n-1$, then

$$
E\left[C_{i, n} \mid \mathscr{F}_{n-1}\right]=C_{i, n+1-i} f_{n+1-i} f_{n-i} \ldots f_{n-1}
$$

So that the year $n$ reserve needed for claims incurred in $i$ is

$$
R_{i}=E\left[C_{i, n} \mid \mathscr{F}_{n-1}\right]-C_{i, n+1-i}
$$

This is rewritten as

$$
R_{i}=C_{i, n+1-i}\left(f_{n+1-i} f_{n-i} \ldots f_{n-1}-1\right)
$$

Hence if we can forecast the pricing kernel $f_{(\cdot)}$ then we can forecast reserves because

$$
\hat{R}_{i}=C_{i, n+1-i}\left(\hat{f}_{n+1-i} \hat{f}_{n-i} \ldots \hat{f}_{n-1}-1\right)
$$

\section{Stochastic Claim Development}

\subsection{Detrended cumulative claims}

First, we start with a nonparametric model introduced by Mack (1994) which focused on the first two moments of an unknown distribution. In particular, Mack proposed the variance of claims reserves as a measure of their risk. We extend that model to detrended claims and show how it justifies stochastic claims modeling. A succinct description of that procedure follows.

Assumption 3.1. Detrended claims are stochastic.

If we believe that claims have a linear trend then we run the regression

$$
C_{i, j}=a_{0}+a_{1} j+\tilde{C}_{i, j}, \quad j=1,2, \ldots, n
$$

where $a_{0}$ and $a_{1}$ are constants, and use the residuals $\tilde{C}_{i, j}$ as our detrended claims. This is the sui generis of the Wold decomposition Theorem 4.1, infra. By definition, detrended claims are fluctuations around a trend. So they are "difference stationary". These fluctuations may be due to systematic factors like accounting and or "seasonal" deadlines for reporting. Mean reversion implies that the unconditional expected value $E\left[\tilde{C}_{i, j}\right]=0$. However, at some point in time, developed 
claims are exhausted so a final payment is made, and they cease to grow so the series is flattened and "killed". Therefore, the trend must reflect that scenario of diminishing growth. See e.g., (Box et al., 1994, pg. 359). Among other things, (De Jong, 2006, pg. 29) used a log link ratio parametrization to address claims growth issues. Thus, an admissible parametrization for our model is

$$
\begin{aligned}
C_{i, j} & =\left(1-\exp ^{-(j-i)}\right) C_{i, \infty}+\tilde{C}_{i, j} \\
\mathrm{P}-\lim _{j \rightarrow \infty} \tilde{C}_{i, j} & =0
\end{aligned}
$$

(Wright, 1990, pg. 682) also presented a Box-Cox type transformation for parametrizing trends in cumulative claims. In which case we could run the regression

$$
C_{i, j}=k(j-i)^{\lambda} C_{i, \infty}+\tilde{C}_{i, j}
$$

where $j-i$ is the delay ${ }^{5}$ in claims payment, $k$ is a constant, and $\lambda$ is a shape parameter.

In the context of Equation 2.3 a detrended claims model imples

$$
\log C_{i, j+1}=\log f_{j}+\log C_{i, j}
$$

This implies that

$$
\log f_{j}=\log C_{i, j+1}-\log C_{i, j}=r_{j}
$$

where $r_{j}$ is the growth rate for claims developed in period $j$ and we can write

$$
f_{j}=\exp \left(r_{j}\right)
$$

This is the basis for De Jong (2006) parametrization. Perhaps, most important for the purpose of this paper, it implies that the pricing kernel $f_{j}$ has exponential growth.

\subsection{Unobservable pricing kernel or link ratio function}

Let $\mathscr{F}_{j}$ be the information set available to the actuary at time $j$. Further, let $f_{j}$ be an unobservable "link ratio function" that "links" claims across time.

\footnotetext{
${ }^{5}$ (Wright, 1990, pg. 681) modeled delay as a continuous random variable. For practical purposes that variable is discrete. See e.g., shot noise process reported in (Küppelberg and Severin, 2001, pg. 1) when claims arrival is an inhomogenous Poisson process,
} 
The behavior of this ratio is critical to our understanding of claims behavior. See e.g.,(Bardis et al., 2008, pg. 2); (Behncke, 2000, pg. 245); (De Jong, 2006, pg. 29); (Mack, 1994, pg. 111). According to (Mack, 1994, pg. 109) given period $j-1$ claims, the conditional expected value for claims developed in period $j$ is

$$
E\left[C_{i, j+1} \mid \mathscr{F}_{j}\right]=f_{j} C_{i, j}
$$

Under that set up, the pricing kernel $f_{j}$ is an unobservable period $j$ link ratio function that needs to be forecast at time $i \leq j$. In fact, as shown below, the proposed claims ladder model proceeds under conditions of risk ("known" probability distribution) and uncertainty (unknown probability distribution) for several variables. It can be shown, see e.g., Mack (1994b, pp. 105) that

$$
\begin{aligned}
C_{i, J} & =C_{i, J+1-i} f_{J+1-i} \ldots f_{J-1}, \quad 2 \leq i \leq J \\
f_{j} & =\frac{\sum_{i=1}^{I-j} C_{i, j+1}}{\left(\sum_{i=1}^{I-j} C_{i, j}\right.}, \quad 1 \leq j \leq I-1
\end{aligned}
$$

\section{Econometric specification of stochastic claims risk}

(Mack, 1994, pg. 111) rewrites the link ratio above as

$$
\begin{aligned}
f_{j} & =\frac{\sum_{i=1}^{I-j} C_{i, j}}{\sum_{i=1}^{I-j} C_{i, j}} \frac{C_{i, j+1}}{C_{i, j}} \\
& =\Sigma_{i=1}^{I-j} w_{i, j} \frac{C_{i, j+1}}{C_{i, j}}
\end{aligned}
$$

Furthermore, he let

$$
\operatorname{Var}\left(\frac{C_{i, j+1}}{C_{i, j}} \mid \mathscr{F}_{j}\right)=E\left[\left\{\frac{C_{i, j+1}-E\left[C_{i, j+1}\right]}{C_{i, j}}\right\}^{2} \mid \mathscr{F}_{j}\right]=\frac{\alpha_{i}^{2}}{C_{i, j}}
$$

which can be rewritten as

$$
\operatorname{Var}\left\{C_{i, j+1} \mid \mathscr{F}_{j}\right\}=C_{i, j} \alpha_{i}^{2}
$$

By definition, this is functionally equivalent to Engle's (1982) ARCH specification, for fluctuations $\tilde{C}_{i, j}$ around a trend, as follows. Let $\xi_{j}$ be the unobservable 
innovation in detrended claims, such that $\operatorname{Var}\left\{\xi_{i}\right\}=\alpha_{i}^{2}$, and write

$$
\tilde{C}_{i, j+1}=\sqrt{\left|\tilde{C}_{i, j}\right|} \xi_{j}
$$

So that unconditionally

$$
E\left[\tilde{C}_{i, j+1}\right]=\sqrt{\left|\tilde{C}_{i, j}\right|} E\left[\xi_{j}\right]=0
$$

Since $E\left[\tilde{C}_{i, j+1}\right]=0$ by construction, this implies that

$$
E\left[\xi_{j}\right]=0
$$

Undeniably, the conditional claims process is stochastic by virtue of being a function of $\xi$-innovations. That is

$$
E\left[\tilde{C}_{i, j+1} \mid \mathscr{F}_{j}\right]=\sqrt{\left|\tilde{C}_{i, j}\right|} \xi_{j}
$$

An earlier paper by Taylor and Ashe (1983) used a tangentially related parametrization as follows

$$
\tilde{C}_{i, j+1}=\mu_{j}+\sigma_{j} \varepsilon_{i, j}
$$

with mean $\left(\mu_{j}\right)$ and variance $\left(\sigma_{j}^{2}\right)$ related only to development year. Thus, if $E\left[\mu_{j}\right]=0$ unconditionally, that model is related. For what follows, we need to introduce

Theorem 4.1 (Wold Decomposition Theorem). Let $\xi(t, \omega)$ be a stationary sequence for $t=0, \pm 1, \pm 2, \ldots$, and let $H_{\xi}$ be the closed linear hull, in $L^{2}(\Omega, \mathscr{F}, \mathbb{F}, P)$, generated by $\xi$. Furthermore, let $H_{\xi}(t)$ be the closed linear hull generated by $\xi$ for $n \leq t$. Let $H_{\xi}^{S}(t)=\cap_{t} H_{\xi}(t) \subset \mathbb{F}$. Then an arbitrary sequence $\xi(t, \omega) \in$ $L^{2}(\Omega, \mathscr{F}, \mathbb{F}, P)$ has a unique decomposition of the form

$$
\xi(t, \omega)=\xi_{S}(t)+\eta(t, \omega)
$$

where $\xi$ and $\eta$ are uncorrelated sequences that are subordinate to $\xi(t, \omega), \xi_{S}(t)$ is deterministic, and $\eta(t, \omega)$ is a $M A(\infty)$ process.

Proof. See (Brockwell and Davis, 1987, pg. 180) and (Gikhman and Skorokhod, 1969, pg. 243). 
Because $\lim _{j \rightarrow \infty} f_{j}=1$ we can write $f_{j}=1+u_{j}$ where P- $\lim _{j} u_{j}=0$. Therefore, $f_{j}$ has a Wold decomposition. See section subsubsection 4.1.1, infra. That is, it can be represented as a $\mathrm{MA}(\infty)$ process. Specifically, since the link function $f_{j}$ is unobservable, let it be measured with error given by $\eta_{j}$. So the actuary observes

$$
\tilde{f}_{j}=f_{j}+\eta_{j}
$$

and the unconditional claim developed in period $j+1$ is now

$$
\tilde{C}_{i, j+1}=f_{j} \tilde{C}_{i, j}+\eta_{j} \tilde{C}_{i, j}
$$

In which case the conditional variance is

$$
\begin{aligned}
\operatorname{Var}\left\{\tilde{C}_{i, j+1} \mid \mathscr{F}_{j}\right\} & =E\left[\left\{\tilde{C}_{i, j+1}-E\left[\tilde{C}_{i, j+1} \mid \mathscr{F}_{j}\right]\right\}^{2}\right] \\
& =\tilde{C}_{i, j}^{2} \operatorname{Var}\left(\eta_{j}\right)=\tilde{C}_{i, j}^{2} \sigma_{\eta_{j}}^{2}
\end{aligned}
$$

Let

$$
\varepsilon_{i, j}=\sqrt{\left|\tilde{C}_{i, j}\right|} \eta_{j}
$$

So that

$$
\operatorname{Var}\left(\varepsilon_{i, j}\right)=\left|\tilde{C}_{i, j}\right| \sigma_{\eta_{j}}^{2}
$$

This implies that we can write

$$
\tilde{C}_{i, j+1}=\sqrt{\left|\tilde{C}_{i, j}\right|} \varepsilon_{i, j}=\tilde{C}_{i, j} \eta_{j}
$$

It is precisely at this point that (Engle, 1982, pg. 988) realized that that autoregressive specification could lead to a variance of zero or infinity, and he suggested the autoregressive conditional heteroskedasticity (ARCH) correction

$$
\begin{aligned}
& \tilde{C}_{i, j+1}=\eta_{j} \sqrt{\sigma_{\tilde{C}_{i, j+1}}^{2}} \\
& \sigma_{\tilde{C}_{i, j+1}}^{2}=\theta_{0}+\theta_{1} \tilde{C}_{i, j}^{2}
\end{aligned}
$$

with the proviso that, unconditionally, $E\left[\eta_{j}\right]=0$ and $\operatorname{Var}\left(\eta_{j}\right)=1$. It should be noted that the foregoing specification handles negative values for incremental claims through the sign of $\eta_{j}$. Thereby, correcting a defect in Mack (1994). 
See (Verrall, 2000, pg. 97). Thus, we have just proven the following

Theorem 4.2 (ARCH in Detrended Stochastic Claims). . Let $\tilde{C}_{i, j}$ be a detrended claim incurred at time $i$ and developed during period $j$. Let $\mathscr{F}_{j}$ be the information set available to the actuary at time $j$, and $f_{j}$ be an unobservable function that links claims in periods $i$ and $j$ such that $\tilde{C}_{i, j+1}=f_{j} \tilde{C}_{i, j}$. Let the conditional variance of claims developed in period $j+1$ be

$$
\operatorname{Var}\left(\tilde{C}_{i, j+1} \mid \mathscr{F}_{j}\right)=\tilde{C}_{i, j} \alpha_{j}^{2}
$$

Suppose that the actuary observes a link function $\tilde{f}_{j}=f_{j}+\eta_{j}$ with unobservable measurement $\eta_{j}$ and link $f_{j}$. Let $E\left[\eta_{j}\right]=0$ and $\operatorname{Var}\left(\eta_{j}\right)=1$. Then detrended claims follows an ARCH process

$$
\begin{gathered}
\tilde{C}_{i, j+1}=\eta_{j} \sqrt{\sigma_{\tilde{C}_{i, j}}^{2}} \\
\sigma_{\tilde{C}_{i, j+1}}^{2}=\theta_{0}+\theta_{1} \tilde{C}_{i, j}^{2}
\end{gathered}
$$

Remark 4.1. This Theorem was derived by extending the Mack (1994) model to detrended claims, and using a fairly standard signal-noise parametrization for the link ratio function. Thus, detrended stochastic claims modeled according to Mack (1994), follows an ARCH process with innovations that depend on claims in the development year. However, Mack did not derive an ARCH specification as we do here.

At Engle's suggestion, Bollerslev proposed a more parsimonious model to mitigate the long lag structure encountered in ARCH models in practice. See (Bollerslev, 1986, pp. 307, 308). Instead of the ARCH process, Bollerslev introduced a Generalized ARCH process which, in the context of our detrended claims process, implies the following

Corollary 4.3 (GARCH(1,1) Detrended Claims Process). . Let $\eta_{j+1}$, the measurement error in observed link function for claims developed during period $j$, be distributed with mean zero and variance $\operatorname{Var}\left(\tilde{C}_{j+1} \mid \mathscr{F}_{j}\right)=\sigma_{\tilde{C}_{i, j}}^{2}$. Then a $\operatorname{GARCH}(1,1)$ process is admissible for evolution of the dynamics of detrended claims. In particular,

$$
\sigma_{\tilde{C}_{i, j+1}}^{2}=\alpha_{1} \varepsilon_{i, j}^{2}+\beta_{1} \sigma_{\tilde{C}_{i, j}}^{2}
$$


Where

$$
\alpha_{1}+\beta_{1}<1
$$

Remark 4.2. By definition in Equation $4.15, \varepsilon_{i, j}$ is a convex function of $\tilde{C}_{i, j}$. Furthermore, the quantity $\varepsilon_{i, j}^{2}=\left|\tilde{C}_{i, j}\right| \eta_{j}^{2}$ reflects the impact of innovations for claims developed in period $j$.

Definition 4.1 (Risk factor exposure). Let $\varepsilon_{i, j}$ be innovations in stochastic claims, and $\sigma_{\tilde{C}_{i, j}}^{2}$ be a measure of stochastic risk. So that in Equation 4.20 stochastic risk at time $j+1$ is a function those two risk factors. Then

A. $\alpha_{1}$ is exposure to innovations in developed claims; and

B. $\beta_{1}$ is exposure to adaptive claims risk.

In what follows we need the following theorem.

Theorem 4.4 (Convergence of Types). Let $\triangle$ connote MLE for a given parameter and derived residual. So that $\stackrel{\Delta}{\alpha}_{1}, \vec{\beta}_{1}$ are MLE for $\alpha_{1}$ and $\beta_{1}$ in the $\operatorname{GARCH}(1,1)$ process

$$
\sigma_{\tilde{C}_{i, j+1}}^{2}=\alpha_{1} \varepsilon_{i, j}^{2}+\beta_{1} \sigma_{\tilde{C}_{i, j}}^{2}
$$

Furthermore, let

$$
P-\lim _{j \rightarrow \infty} \stackrel{\Delta}{\sigma}_{\tilde{C}_{i, j+1}}^{2}=\frac{\sigma_{\tilde{C}_{i}}^{2}}{1-\alpha_{1}-\beta_{1}}
$$

Then for any continuous function $g \in C^{2}(\mathbb{R})$ we have

$$
P-\lim g\left(\stackrel{\Delta}{\alpha}_{1}, \stackrel{\Delta}{\beta}_{1}\right)=g\left(\alpha_{1}, \beta_{1}\right)
$$

Proof. See (Bollerslev, 1986, Thm. 1 and 2 pp. 310-311) and "convergence of types theorem" in (Durrett, 2005, pg. 156).

It is clear from Equation 4.20 that we can write innovations in stochastic claims as a function of the risk factor exposures defined in 4.1. In particular

$$
\stackrel{\Delta}{\varepsilon}_{i, j}=\frac{\stackrel{\Delta}{\sigma}_{i, j+1}^{2}-\stackrel{\Delta}{\beta}_{1} \stackrel{\Delta}{\sigma}_{i, j}^{2}}{\stackrel{\Delta}{\alpha}_{1}}
$$


On average, MLE estimates of $\alpha_{1}$ and $\beta_{1}$ are consistent and efficient. However, an empirical regularity for $\operatorname{GARCH}(1,1)$ is that $\stackrel{\Delta}{\beta}_{1} \gg \stackrel{\Delta}{\alpha}_{1}$. That is, stochastic claims risk exposure portends persistent claims risk, while exposure to innovations in developed claims is suggests that innovations are comparatively transient. See e.g., (Davidson and MacKinnon, 2004, pg. 579); (Shephard, 1996, pg. 13). Thus, we have the following

Proposition 4.5. Let $\varepsilon_{i, j}$ be the innovation in claims incurred at time $i$ and developed in period $j-1$, and $\sigma_{i, j}$ be the corresponding claims risk. Suppose that claims risk dynamics follows a GARCH $(1,1)$ process so that

$$
\stackrel{\Delta}{\varepsilon}_{i, j}=\frac{\stackrel{\Delta}{\sigma}_{i, j+1}^{2}-\stackrel{\Delta}{\beta}_{1} \stackrel{\Delta}{\sigma}_{i, j}^{2}}{\dot{\Delta}_{1}}
$$

Then claims risk exposure $\alpha_{1}$ portends persistent claims risk, and $\beta_{1}$-the exposure to innovations, portends transient shocks to claims risk.

Proof. See Theorem 4.4 .

\subsection{Pricing kernel estimator}

The foregoing analysis shows that $\mathrm{ARCH}$ and $\mathrm{GARCH}$ are admissible models for claim fluctuation around trend. However, these fluctuations must decay to reflect long run convergence to developed claims. See e.g., Appendix A, Fig. 1: Average Cumulative Percentage of Claims Paid By Development Year in Nelson (2000). Specifically, we claim that $\tilde{C}_{i, j}$ is well defined by proving that

$$
\tilde{C}_{i, j+1}=\sqrt{\left|\tilde{C}_{i, j}\right|} \varepsilon_{i, j}
$$

is an admissible decay model for claim fluctuations. See e.g., (Wilkie, 1995, pg 928). See also, (Engle, 2004, pg. 407). Let

$$
\tilde{C}_{i, 1}=\sqrt{\left|\tilde{C}_{i, 0}\right|} \varepsilon_{i, 0}
$$

Then, by recursion, we get

$$
\tilde{C}_{i, k}=\varepsilon_{i, k-1}\left|\varepsilon_{i, k-2}\right|^{-2^{-1}} \ldots\left|\varepsilon_{i, 0}\right|^{-2^{-k+1}}\left|\tilde{C}_{i, 0}\right|^{-2^{-k}}
$$


In which case,

$$
\lim _{k \rightarrow \infty} \tilde{C}_{i, k}=\lim _{k \rightarrow \infty} \varepsilon_{i, k-1}\left|\varepsilon_{i, k-2}\right|^{-2^{-1}} \ldots\left|\varepsilon_{i, 0}\right|^{-2^{-k+1}}\left|\tilde{C}_{i, 0}\right|^{-2^{-k}}=0
$$

assuming that the $\varepsilon$-fluctuations are such that they dampen to zero. This is a pseudo Kalman filter result because past error is used for forecasting. See (Box et al., 1994, pg. 165). Because $|\varepsilon|<1$, the index suggests that these are the "stochastic claims inflation" factor in (Kremer, 1999, pg. 106). So the derived fluctuations $\tilde{C}_{i, k}$ decay and

$$
\mathrm{P}-\lim _{k \rightarrow \infty} \tilde{C}_{i, k}=0
$$

Thus we have just proved the following

Theorem 4.6 (Pricing Kernel Estimator). . Let $\tilde{C}_{i, j}$ be the detrended stochastic claim incurred at time I but not developed until period $j$. Let $f_{j}$ be the unobservable link ratio function for claims developed in period $j$, and $\eta_{j}$ be concommittant measurement error. So that the actuary observes $\tilde{f}_{j}=f_{j}+\eta_{j}$. Then the stochastic claims inflation factor, i.e. pricing kernel, for claims claims incurred in period $i$ and developed at time $j$ is given by

$$
\varepsilon_{i, j}^{-1}=\frac{1}{\sqrt{\left|\tilde{C}_{i, j}\right|} \eta_{j}}
$$

Because $\varepsilon_{i, j}$ and $\eta_{j}$ are estimable from ARCH and or GARCH diagnostics we get cross validation for $f_{j}$ by extrapolating $\hat{f}_{j}=\tilde{f}_{j}-\Delta_{j}$ by virtue of Theorem 4.4. It is enough to claim that estimation of pricing kernel noise is given by

$$
\stackrel{\triangle}{\eta}_{j}=\frac{\stackrel{\Delta}{\varepsilon}_{i, j}}{\left|\tilde{C}_{i, j}\right|}
$$

So that the signal to noise ratio for the link ratio function or pricing kernel is

$$
S N R_{\text {ClaimInfl }}=\frac{\stackrel{\Delta}{\sigma}_{\tilde{f}_{j}}^{2}}{\stackrel{\Delta}{\sigma}_{\eta_{j}}^{2}}
$$

If $S N R_{\text {ClaimInfl }}>1$, then our model is picking up the "signal" from the true link 
ratio function. In the actuarial literature the coefficient of variation

$$
\left(\operatorname{SNR}_{\text {ClaimInfl }}^{c v}\right)^{-1}=\left(\frac{\stackrel{\Delta}{\mu}_{f_{j}}}{\vec{\sigma}_{f_{j}}}\right)^{-1}
$$

is used as an alternative to Equation 4.28. See (Shapland, 2007, pp. 130-131).

\subsubsection{Wold decomposition of pricing kernel}

According to Wold Dcomposition Theorem 4.1 if $S N R<1$, then $f_{j}$ has a long MA representation for trend. If $S N R>1$, then the deterministic component dominates and the MA representation for trend in short. See (Mills and Markellos, 2008, pg. 118).

Consider the following argument. Let

$$
\begin{aligned}
& f_{j}=1+u_{j} \\
& u_{j}=\theta u_{j-1}+v_{j}, \quad|\theta|<1
\end{aligned}
$$

Suppose that $\eta_{j}$ is white noise, so that

$$
\eta_{j}=\eta_{j-1}+e_{j}
$$

Then

$$
\begin{aligned}
\Delta \tilde{f}_{j} & =\Delta f_{j}+\Delta \eta_{j} \\
& =(1-\theta L)^{-1}(1-L) v_{j}+e_{j}
\end{aligned}
$$

where $\Delta$ is a difference operator, and $L$ is a lag operator. Under Wold decomposition $\Delta \tilde{f}_{j}$ is difference stationary. Thus we have the signal

$$
z_{j}=(1-\theta L)^{-1}(1-L) v_{j}
$$

and noise $e_{j}$. Undeniably, $z_{j}$ has a moving average (MA) representation. Thus, the "new" SNR is

$$
S N R=\frac{\sigma_{z}^{2}}{\sigma_{e}^{2}}=\left(1+\frac{2 \theta^{2}}{1-\theta}\right) \frac{\sigma_{v}^{2}}{\sigma_{e}^{2}}
$$

The behavior of $\theta$ determines the magnitude of $S N R$. As long as $\theta$ is in the 
unit circle SNR will be inflated, i.e greater than 1. In particular, if $0<\theta<1$ then the signal should be strong. In any case, the decay hypothesis is suppported by Wold decomposition. For instance, Equation 4.36 satisfies (Shapland, 2007, Concept 1.pg. 130) which reads, in pertinent part:

For each (accident, policy, or report) year, the coefficient of variation (standard error as a percentage of estimated liabilities) should be the largest for the oldest (earliest) year and will, generally, get smaller when compared to more and more recent years.

Evidently, the coefficient of variation (CV) is large when the SNR is small since $S N R=(C V)^{-1}$. Which implies that the moving average term is relatively dominant, i.e., the series is getting longer.

\section{Insurance accounting and performance measurement}

According to (Nelson, 2000, pg. 8) "The primary input used by insurers in developing rates, and by regulators in evaluating those rates, is the loss reserve estimate". Furthermore

Statutory accounting practices require insurers to charge claim losses to operations in the period in which they are incurred, even though many years may elapse before the claims are actually paid. As a result, insurers must estimate the amount required to settle the incurred but unpaid claims, including direct expenses associated with the claims settlement process (e.g., litigation costs). This liability, known as the loss reserve, should be reported at nominal value in the insurers statutory annual statement filed with state insurance regulators. 2 The loss reserve is revised as new information becomes available, until all claims are settled and total incurred losses are known with certainty.

(Nelson, 2000, pg. 4). For instance, the rate making process provides for a (1) loss ratio method (LRM) or (2) a pure premium method (PPM) defined as follows

$$
\begin{gathered}
L R M_{i, j}=\frac{\text { Paid_Losses }_{i, j}+C_{i, j}}{\text { Earned_Premium }_{i, j}} \\
P P M_{i, j}=\frac{E\left[C_{i, j}\right]}{\text { Exposure_Units }_{i, j}}
\end{gathered}
$$


Additionally, according to (Ward and Lee, 2002, pp. 120-121) the Risk-adjusted Return on Capital (RAROC) for a non-life insurance company is

$$
R A R O C_{i, j}=\frac{U W_{i, j}+I C_{i, j}+C B_{i, j}}{E C_{i, j}}
$$

where

$$
\begin{aligned}
\mathrm{UW} & =\text { underwriting result } \\
\mathrm{IC} & =\text { investment credit } \\
\mathrm{CB} & =\text { capital benefit } \\
\mathrm{EC} & =\text { economic capital }
\end{aligned}
$$

Of interest to us in that formula is the underwriting result which is adjusted to account for changes in company experience

$$
\text { AdjUW }=U W-\Delta C_{i, j}+\text { Overhead }- \text { OneTimeCharges }
$$

where $\Delta C_{i, j}$ is change in reserves. To avoid overloading the paper, we will not go into details about the ramifications of the foregoing formulae since the cited references covers them thoroughly. Nonetheless, it is clear that the variable $C_{i, j}$ plays a prominent role in both statutory accounting requirements and computation of risk adjusted return on capital ${ }^{6}$. Therefore, those performace measures of the company are affected by claims forecast which, in our model, are determined by risk exposure factors.

\section{Conclusion}

This paper provided a theoretical model of claims reserve forecast based on the Wold decomposition, and workhorse $\operatorname{GARCH}(1,1)$ model in financial econometrics. Diagnostics from that model was used to provide forecasts of the pricing kernel for IBNR claim development, cross validate, and compute signal to noise ratio for unobservable link ratio functions-cross validated by Wold decomposition In practice, that model may be difficult to implement because there may not be enough data points. However, (Nelson, 2000, pg. 8) reported that the average

\footnotetext{
${ }^{6}$ A survey by Graham and Harvey (2001) showed that large companies used the CAPM to compute return on capital (ROC) but small firms did not. So our results may be more applicable to lareg companies.
} 
range for claims development exhaustion is 7-years for Homeowners/Farmowners Multiple Peril (HM) and 20-years for Medical Malpractice (MM). Therefore, the methodology proposed here may be better suited to a monthly or quarterly series of long tail claims. Additionally, we prove that seemingly unrelated specifications for loss link ratio, and claims volatility, are linked by a single risk exposure factor. Because our results were driven by Wold decomposition-of which the $\operatorname{GARCH}(1,1)$ model is a special case-further research in this area includes extension to the panoply of ARCH models, and other time series decomposition models. 


\section{References}

Bardis, E. T., A. Majidi, and D. M. Murphy (2008). Manually Adjustable Link Ratio Model For Reserving. Casualty Actuarial Society E-Forum, 1-15.

Beaver, W. H., M. F. Mc Nichols, and K. K. Nelson (2003). Management of the Loss Reserve Accrual and the Distribution of Earnings in the Property-Casualty Insurance Industry. Journal of Accounting and Economics 35, 347-376.

Behncke, H. (2000). Insurance Mathematics: A European Model, mimeo. Universität Osnabrück - Institut für Mathematik, Germany; available at ftp://ftp.mathematik.uni-osnabrueck.de/pub/osm/versicher.ps.gz.

Bollerslev, T. (1986). Generalized Autoregressive Conditional Heteroskedasticity. Journal of Econometrics 31, 307-327.

Box, G. E. P., G. M. Jenkins, , and G. Riensel (1994). Time Series Forecasting and Control (3rd ed.). New Jersey: Prentice-Hall, Inc.

Brockwell, P. J. and R. A. Davis (1987). Time Series: Theory and Methods. New York, NY: Springer-Verlag.

Calandro, J. and T. J. O`Brein (2004). A User Friendly Introduction to Property and Casualty Claim Reserves. Risk Management and Insurance Review 7(2), 177-187.

Campbell, J. Y., A. W. Lo, and A. C. MacKinlay (1997). The Econometrics of Financial Markets. Princeton, N. J.: Princeton University Press.

Davidson, R. and J. G. MacKinnon (2004). Econometric Theory and Methods. New York: Oxford Univ. Press.

De Jong, P. (2006). Forecasting Runoff Triangles. North American Actuarial Journal 10(2), 28-38.

Durrett, R. (2005). Probability: Theory and Examples (3rd ed.). Belmont, CA: Thompson Brooks-Cole. 
Engle, R. (1982, July). Autoregressive Conditional heteroskedasticity with Estimates of the Variance of United Kingdom Inflation. Econometrica 50(4), 9871008 .

Engle, R. F. (2001). GARCH101: The Use of ARCH/GARCH Models in Applied Econometrics. Journal of Economic Perspectives 15(4), 157-168.

Engle, R. F. (2004). Risk and Volatility: Econometric Models and Financial Practice. Amer. Econ. Rev. 94(3), 405-420.

Gikhman, I. I. and A. V. Skorokhod (1969). Introduction to The Theory of Random Processes (Dover reprint 1996 ed.). Philadelphia, PA: W. B. Saunders.

Graham, J. R. and C. Harvey (2001). The Theory and Practice of Corporate Finance: Evidence From The Field. Journal of Financial Economics 60, 187-243.

Kremer, E. (1999, Oct.). Stochastic Claim Inflation in IBNR. presented at ASTIN Colloquium 1998, Glasgow, Scotland; published in Blatter der DGVFM 24(2):231-238.

Küppelberg, C. and M. Severin (2001). Prediction of Outstanding Claims. Institute of Statistics, Ludwig-Maximilians University in Munich, Paper No. 258, available at http://epub.ub.uni-muenchen.de/.

Mack, T. (1994). Measuring The Variability of Chain Ladder Reserve Estimates. Casualty Actuarial Society Forum 1(1), 101-83.

Mills, T. C. and T. N. Markellos (2008). The Econometric Modeling of Financial Time Series (3rd ed.). New York: Cambridge University Press.

Nelson, K. K. (2000, Jan.). Rate Regulation, Competition, and Loss Reserve Discounting by Property-Casualty Insurers. Accounting Review 75(1), 115-138.

Shapland, M. R. (2007). Loss Reserve Estimation: A Statistical Approach for Determining Reasonableness. Variance:Advancing The Science of Risk 1(1), 120-148.

Shephard, N. (1996). Time Series Models in Econometrics, Finance and Other Fields, Chapter Statistical Aspects of ARCH and Stochastic Volatility, pp. 167. London: Chapman \& Hall. 
Taylor, G. (1977). Separation of Inflation and Other Effects From the Distribution of Non-life Insurance Claim Delays. Astin Bulletin 9, 219-230.

Taylor, G. C. and F. R. Ashe (1983). Second Moments of Estimates of Outstanding Claims. Journal of Econometrics 23(1), 37 - 61.

Verrall, R. J. (2000). An Investigation Into Stochastic Claims Reserving Models and The Claims Ladder Technique. Insurance, Mathematics and Economics 26, 91-98.

Ward, L. S. and D. H. Lee (2002). Practical Application of the Risk-Adjusted Return on Capital Framework. CAS Forum Summer 2002, Dynamic Financial Analysis, available at http://www.casact.org/pubs/forum/02sforum/02sf079.pdf.

Wilkie, A. D. (1995). More On A Stochastic Asset Model For Actuarial Use. British Actuarial Journal 1(5), 777-964.

Wright, T. S. (1990). A Stochastic Method for Claims Reserving in General Insurance. Journal of Institute of Actuaries 117, 677-731. 